PROCEEDINGS OF THE

AMERICAN MATHEMATICAL SOCIETY

Volume 127, Number 11, Pages 3427-3432

S 0002-9939(99)04983-7

Article electronically published on May 6, 1999

\title{
MAXIMAL BENNEQUIN NUMBERS AND KAUFFMAN POLYNOMIALS OF POSITIVE LINKS
}

\author{
TOSHIFUMI TANAKA
}

(Communicated by Ronald A. Fintushel)

\begin{abstract}
By using results of Yamada and of Yokota, concerning link diagrams and link polynomials, we give some relationships between maximal Bennequin numbers and Kauffman polynomials of positive links.
\end{abstract}

\section{INTRODUCTION}

A contact structure in a smooth 3 -dimensional manifold $M$ is a global differential 1-form $\eta$ such that $\eta \wedge(d \eta) \neq 0$ everywhere on $M$. A contact distribution is the subbundle of $T M$ on which the contact structure vanishes. The standard contact structure in 3-space is the differential 1-form $d z-y d x$. A Legendrian link is a $C^{\infty}$ embedding of disjoint circles in 3-space with the standard contact structure, which are everywhere tangent to the contact distribution. We call the composition of a Legendrian link $L$ and the $(x, z)$-projection of 3 -space, the front of $L$. The front of a Legendrian link may have cusps because the fibers of the contact distribution are parallel to the $y$-axis. Generically, the only singularities of a front are cusps and transverse double points [4]. We assume that all fronts are generic in this paper. A Legendrian isotopy between Legendrian links $L_{0}$ and $L_{1}$ is an ambient isotopy between $L_{0}$ and $L_{1}$, with each level Legendrian. It is known that two Legendrian links are Legendrian isotopic if and only if their fronts are related by a sequence of the Legendrian version of Reidemeister moves in Figure 1.

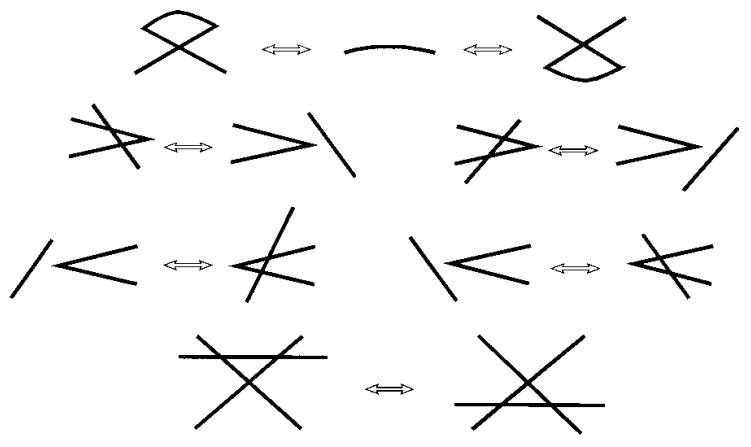

FiguRe 1

Received by the editors September 27, 1997 and, in revised form, February 6, 1998. 1991 Mathematics Subject Classification. Primary 57M50, 57M25.

Key words and phrases. Positive links, Bennequin number, Kauffman polynomial. 
The Bennequin number denoted by $\beta(L)$ of an oriented Legendrian link $L$ is defined, using the $(x, z)$-projection by the following equation:

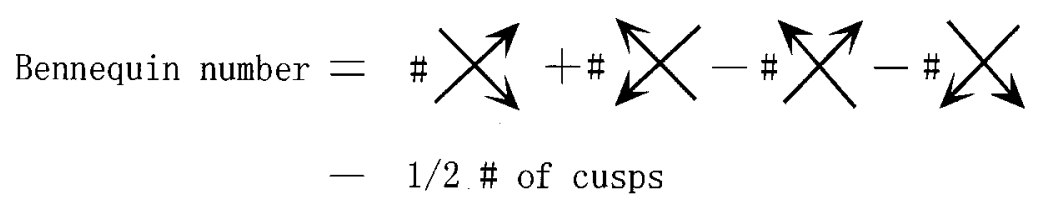

FiguRE 2

The Bennequin number is a Legendrian isotopy invariant of Legendrian links.

Let $D$ be a diagram of an oriented link $L$ and $\omega(D)$ be the writhe of $D$. The Kauffman polynomial $F_{(x, y)}(L) \in \mathbf{Z}\left[x^{ \pm}, y^{ \pm}\right]$is defined by $a^{-\omega(D)} \bigwedge_{(x, y)}(D)$, where $\bigwedge_{(x, y)}(D)$ is a regular isotopy invariant of diagrams of $L$ with the properties in Figure 3. The Kauffman polynomial is a topological invariant of oriented links.

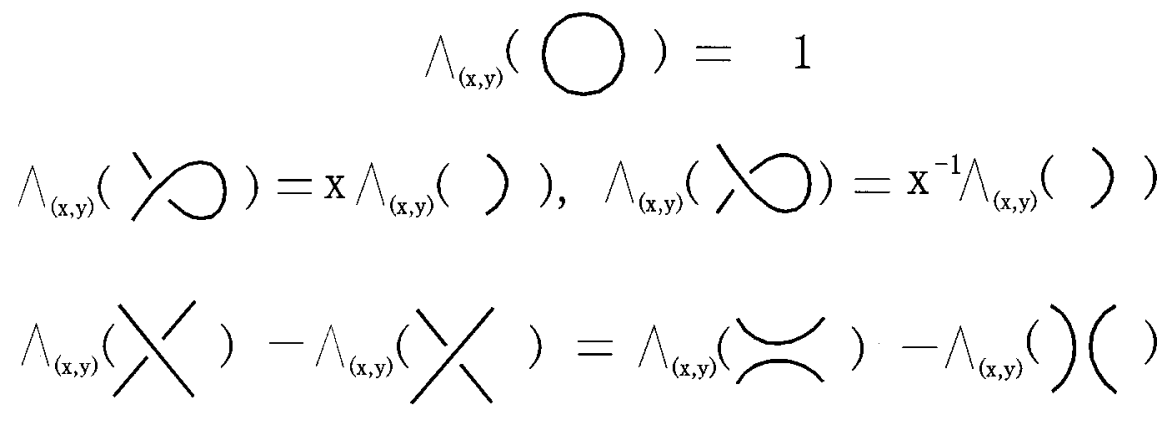

FIGURE 3

In this paper, we consider the relationship between the degree of the Kauffman polynomial of an oriented link $L$ and the maximal value, denoted by $B(L)$, of the Bennequin numbers for Legendrian links ambient isotopic to $L$. An upper bound on $B(L)$ in terms of the Kauffman polynomial is given in [7], [8] as follows.

Theorem 1. Let $L$ be a Legendrian link. Then the following inequality holds:

$$
B(L)<-\max -\operatorname{deg}_{x} F_{(x, y)}(L) .
$$

Remark. Our proof of Theorem 1 seems different from that given in [7], [8].

By using Theorem 1 (see Figure 9), we can show that almost all the knots $L$ of 9 or fewer crossings in the knot table of Rolfsen [1] satisfy the equality

$$
B(L)+1=-\max -\operatorname{deg}_{x} F_{(x, y)}(L) .
$$

So we consider the following problem.

Problem. Does the above equality hold for any oriented link?

An oriented link is said to be positive if $L$ has a diagram with no negative crossings. Our main result is the following: 
Theorem 2. Let $L$ be a positive link and let $D$ be any positive diagram of $L$. Then the following equality holds:

$$
B(L)+1=\omega(D)-S(D)+1=-\max -\operatorname{deg}_{x} F_{(x, y)}(L),
$$

where $\omega(D)$ and $S(D)$ are the writhe and number of Seifert circles of $D$ respectively.

Remark. Rudolph defined the above invariant for any knot [6]. He shows that if a knot $K$ has non-negative Bennequin invariant, then $K$ is not slice. He also shows that if $K$ is a non-trivial strongly quasipositive knot [6], then $B(K) \geq 0$. From the fact above and Theorem 1, we know that any non-trivial strongly quasipositive knot has negative degree in $x$ of the Kauffman polynomial.

\section{Proofs}

Proof of Theorem 1. Let $L$ be a Legendrian link in $\mathbf{R}^{3}$. We denote the front of $L$ by $F_{L}$. We can convert $F_{L}$ into a diagram of the link with the same (topological) isotopy type by using the local deformations as in Figure 4. (Round the cusps and make the strand with the smaller slope overcross at each double point.) We denote the resultant diagram by $D_{L}$. We now flatten $D_{L}$ in a small neighberhood of each crossing as in Figure 5 . We denote the resultant diagram by $\widehat{D_{L}}$.

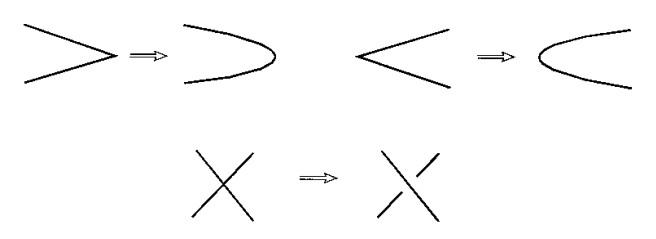

Figure 4

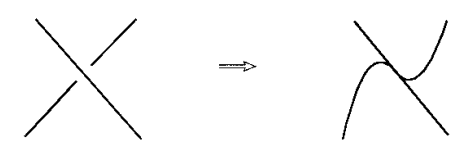

Figure 5

Let $\psi$ be a height function from the $(x, z)$-plane to the $x$-axis. Note that $\widehat{D_{L}}$ lies in general position relative to $\psi$. In fact, there is no local maximal point or minimal point in $F_{L}-$ \{small neighborhood of a cusp $\}$ because $F_{L}$ has no vertical tangents. Thus we can assume that a point $p$ is a local maximal (or local minimal) point in $\widehat{D_{L}}$ if and only if $p$ corresponds to the cusp in $F_{L}$. Let $C\left(F_{L}\right)$ be the number of cusps in $F_{L}$ and $b\left(\widehat{D_{L}}\right)$ be the number of local maxima in $\widehat{D_{L}}$ with respect to $\psi$. Now we obtain that $1 / 2 C\left(F_{L}\right)=b\left(\widehat{D_{L}}\right)$.

Then by using Lemma 1 in [5], we know that

$$
\max -\operatorname{deg}_{x} \bigwedge_{(x, y)}\left(D_{L}\right) \leq 1 / 2 C\left(F_{L}\right)-1
$$


So,

$$
\begin{aligned}
-\max _{-} \operatorname{deg}_{x} F_{(x, y)}(L) & =\omega\left(D_{L}\right)-\max -\operatorname{deg}_{x} \bigwedge_{(x, y)}(L) \\
& \geq \omega\left(D_{L}\right)-1 / 2 C\left(F_{L}\right)+1 \\
& =\beta(L)+1>\beta(L) .
\end{aligned}
$$

This completes the proof.

Proof of Theorem 2. It is known that, by using so-called "bunching deformations" [3] as in [5] (see Figure 7) that every positive link diagram of a link $L$ can be transformed into a positive diagram $D_{L}$ of $L$ which satisfies the following properties:

i) each crossing is oriented downward relative to $\psi$ as in Figure 6;

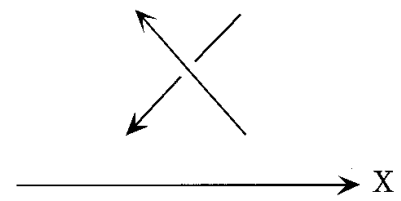

Figure 6

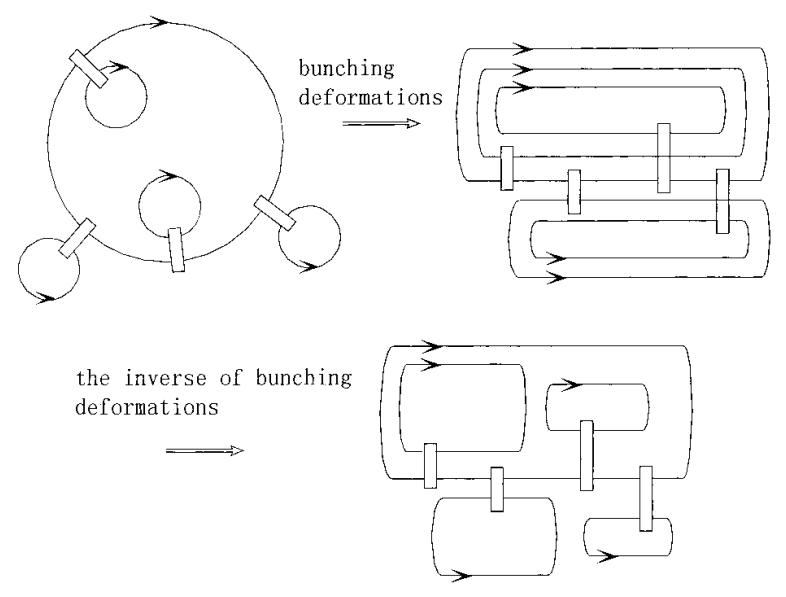

FiguRE 7

ii) each Seifert circle has exactly 1 local maximum and 1 local minimum relative to $\psi$.

We now construct the front $D_{+}$of a Legendrian link $L_{+}$which has the same topological isotopy type as in Figure 8. (Make the neighborhoods of local maxima and local minima into cusps and reinsert the crossings which connected Seifert circles.)

Let $S\left(D_{+}\right)$be the number of Seifert circles in $D_{+}$. Then by using the equality concerning the Kauffman polynomial below the Main Theorem of [5], we obtain that

$$
\begin{aligned}
\max -\operatorname{deg}_{x} F_{(x, y)}(L) & =-\omega\left(D_{+}\right)+S\left(D_{+}\right)-1 \\
& =-\omega\left(D_{+}\right)+1 / 2 C\left(D_{+}\right)-1=-\beta\left(L_{+}\right)-1 .
\end{aligned}
$$




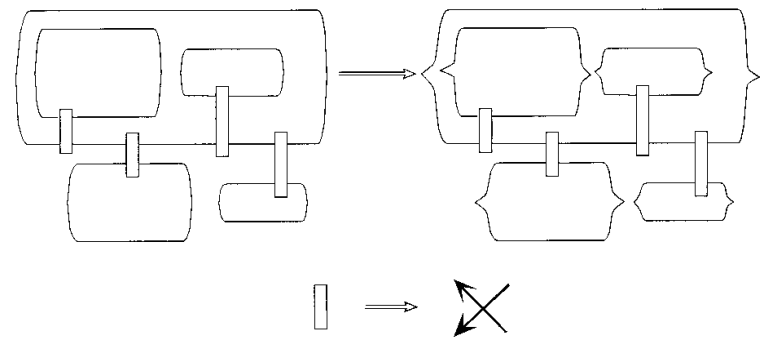

FiguRE 8

Now the result follows from Theorem 1 at once.

Let $n(K)$ be the max-degree in $x$ of the Kauffman polynomial of a knot $K$. We have calculated the Bennequin invariants of the knots with 9 or fewer crossings; the results are tabulated in Figure 9. (All knots are oriented as in [2]. We know the marked knots are positive. The round bracket $(x)$ means that the number is at least $x$.)

\begin{tabular}{|r|r|r|l|r|r|l|r|r|r|r|r|r|r|r|}
\hline knot & $\mathrm{B}(\mathrm{K})$ & $-\mathrm{n}(\mathrm{K})$ & $6_{2}$ & -1 & 0 & $7_{5}{ }^{*}$ & 3 & 4 & $8_{4}$ & -7 & -6 & $8_{10}$ & -2 & -1 \\
\hline $3_{1}{ }^{*}$ & 1 & 2 & $6_{3}$ & -4 & -3 & $7_{6}$ & -1 & 0 & $8_{5}$ & 1 & 2 & $8_{11}$ & -1 & 0 \\
\hline $4_{1}$ & -3 & -2 & $7_{1}{ }^{*}$ & 5 & 6 & $7_{7}$ & -5 & -4 & $8_{6}$ & -1 & 0 & $8_{12}$ & -5 & -4 \\
\hline $5_{1}{ }^{*}$ & 3 & 4 & $7_{2}$ & -10 & -9 & $8_{1}$ & -7 & -6 & $8_{7}$ & -2 & -1 & $8_{13}$ & -2 & -1 \\
\hline $5_{2}$ & -8 & -7 & $7_{3}{ }^{*}$ & 3 & 4 & $8_{2}$ & -11 & -10 & $8_{8}$ & -6 & -5 & $8_{14}$ & -9 & -8 \\
\hline $6_{1}$ & -5 & -4 & $7_{4}$ & -10 & -9 & $8_{3}$ & -5 & -4 & $8_{9}$ & -5 & -4 & $8_{15}^{*}$ & 3 & 4 \\
\hline
\end{tabular}

\begin{tabular}{|r|r|r|l|r|r|l|r|r|r|r|r|r|r|r|}
\hline $8_{16}$ & -2 & -1 & $9_{1}{ }^{*}$ & 7 & 8 & $9_{7}^{*}$ & 3 & 4 & $9_{13}{ }^{*}$ & 3 & 4 & $9_{19}$ & -6 & -5 \\
\hline $8_{17}$ & -5 & -4 & $9_{2}$ & -12 & -11 & $9_{8}$ & -3 & -2 & $9_{14}$ & -7 & -6 & $9_{20}$ & -12 & -11 \\
\hline $8_{18}$ & -5 & -4 & $9_{3}{ }^{*}$ & 5 & 6 & $9_{9}^{*}$ & 5 & 6 & $9_{15}$ & -10 & -9 & $9_{21}$ & -1 & 0 \\
\hline $8_{19}$ & $(-14)$ & -10 & $9_{4}^{*}$ & 3 & 4 & $9_{10}^{*}$ & 3 & 4 & $9_{16}$ & -16 & -15 & $9_{22}$ & -3 & -2 \\
\hline $8_{20}$ & -2 & -1 & $9_{5}^{*}$ & 1 & 2 & $9_{11}$ & 1 & 2 & $9_{17}$ & -8 & -7 & $9_{23}$ & -14 & -13 \\
\hline $8_{21}$ & $(-1)$ & 2 & $9_{6}^{*}$ & 5 & 6 & $9_{12}$ & -1 & 0 & $9_{18}^{*}$ & 3 & 4 & $9_{24}$ & -5 & -4 \\
\hline
\end{tabular}

\begin{tabular}{|c|c|c|c|c|c|c|c|c|c|c|c|c|}
\hline${ }^{9_{25}}$ & -1 & 0 & $9_{31}$ & -2 & -1 & $9_{37}$ & -5 & -4 & $9_{43}$ & 1 & 2 & \begin{tabular}{l|l|l|}
949 & $(-13)$ & -11 \\
\end{tabular} \\
\hline $9_{26}$ & -2 & -1 & $9_{32}$ & -2 & -1 & $9_{38}^{*}$ & 3 & 4 & $9_{44}$ & -6 & -5 & \\
\hline $9_{27}$ & -5 & -4 & $9_{33}$ & -5 & -4 & $9_{39}$ & -10 & -9 & $9_{45}$ & -10 & -9 & \\
\hline $9_{28}$ & -9 & -8 & $9_{34}$ & -6 & -5 & $9_{40}$ & -2 & -1 & $9_{46}$ & $(-8)$ & -6 & \\
\hline $9_{29}$ & -8 & -7 & $9_{35}$ & -12 & -11 & $9_{41}$ & -7 & -6 & $9_{47}$ & -7 & -6 & \\
\hline $9_{30}$ & -5 & -4 & $9_{36}$ & 1 & 2 & $9_{42}$ & $(-6)$ & -2 & $9_{48}$ & $(-9)$ & -7 & \\
\hline
\end{tabular}

FiguRE 9 


\section{ACKNOWLEDGEMENTS}

The auther would like to thank Professor Mitsuyoshi Kato for his encouragement and he also thanks Professor Yoshiyuki Yokota for his information.

\section{REFERENCES}

1. D. Rolfsen, Knots and links, Publish or Perish, Inc. (1976). MR 58:24236

2. L. H. Kauffman, On knots, Ann. of Math. Studies 115. Princeton Univ. Press (1987). MR 89c: 57005

3. S. Yamada, The minimal number of Seifert circles equals to the braid index of a link, Invent. Math., Vol. 89, (1987). MR 88f:57015

4. J. Swiatkowski, On the isotopy of Legendrian knots, Ann. Glob. Anal. Geom. Vol. 10, pp. 195-207 (1992). MR 93m:57010

5. Y. Yokota, Polynomial invariants of positive links, Topology, Vol. 31, No. 4, pp. 805-811 (1992). MR 93k: 57028

6. L. Rudolph, An obstruction to sliceness via contact geometry and "classical" gauge theory, Invent. Math, Vol. 199, pp. 155-163 (1995). MR 95k:57013

7. D. Fuchs, S. Tabachnikov, Invariants of Legendrian and transverse knots in the standard contact space, Topology, Vol. 36, No. 5, pp. 1025-1053 (1997). CMP 97:11

8. S. Tabachnikov, Estimates for the Bennequin number of Legendrian links from state models for knot polynomials, Math. Res. Let. Vol. 4, pp. 143-156 (1997). CMP 97:08

Graduate School of Mathematics, Kyushu University, Hakozaki 6-10-1, Higashiku, FUKUOKA, 812-8581 JAPAN

E-mail address: ttanaka@math.kyushu-u.ac.jp 